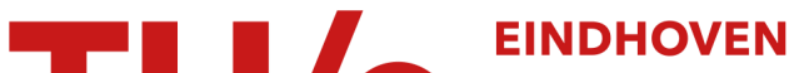 UNIVERSITY OF TECHNOLOGY
}

\section{Universality for distances in power-law random graphs}

\author{
Citation for published version (APA): \\ Hofstad, van der, R. W., \& Hooghiemstra, G. (2008). Universality for distances in power-law random graphs. \\ Journal of Mathematical Physics, 49(12), 125209-1/14. https://doi.org/10.1063/1.2982927
}

DOI:

10.1063/1.2982927

Document status and date:

Published: 01/01/2008

\section{Document Version:}

Publisher's PDF, also known as Version of Record (includes final page, issue and volume numbers)

\section{Please check the document version of this publication:}

- A submitted manuscript is the version of the article upon submission and before peer-review. There can be important differences between the submitted version and the official published version of record. People interested in the research are advised to contact the author for the final version of the publication, or visit the $\mathrm{DOI}$ to the publisher's website.

- The final author version and the galley proof are versions of the publication after peer review.

- The final published version features the final layout of the paper including the volume, issue and page numbers.

Link to publication

\section{General rights}

Copyright and moral rights for the publications made accessible in the public portal are retained by the authors and/or other copyright owners and it is a condition of accessing publications that users recognise and abide by the legal requirements associated with these rights.

- Users may download and print one copy of any publication from the public portal for the purpose of private study or research.

- You may not further distribute the material or use it for any profit-making activity or commercial gain

- You may freely distribute the URL identifying the publication in the public portal.

If the publication is distributed under the terms of Article 25fa of the Dutch Copyright Act, indicated by the "Taverne" license above, please follow below link for the End User Agreement:

www.tue.nl/taverne

Take down policy

If you believe that this document breaches copyright please contact us at:

openaccess@tue.nl

providing details and we will investigate your claim. 


\title{
Universality for distances in power-law random graphs
}

\author{
Remco van der Hofstad ${ }^{1, a)}$ and Gerard Hooghiemstra ${ }^{2, b)}$ \\ ${ }^{1}$ Department of Mathematics and Computer Science, Eindhoven University of Technology, \\ P.O. Box 513, $5600 \mathrm{MB}$ Eindhoven, The Netherlands \\ ${ }^{2}$ DIAM, Delft University of Technology, Mekelweg 4, 2628CD Delft, The Netherlands
}

(Received 30 May 2008; accepted 27 August 2008; published online 12 December 2008)

\begin{abstract}
We survey the recent work on phase transition and distances in various random graph models with general degree sequences. We focus on inhomogeneous random graphs, the configuration model, and affine preferential attachment models, and pay special attention to the setting where these random graphs have a power-law degree sequence. This means that the proportion of vertices with degree $k$ in large graphs is approximately proportional to $k^{-\tau}$ for some $\tau>1$. Since many real networks have been empirically shown to have power-law degree sequences, these random graphs can be seen as more realistic models for real complex networks than classical random graphs such as the Erdős-Rényi random graph. It is often suggested that the behavior of random graphs should have a large amount of universality, meaning, in this case, that random graphs with similar degree sequences share similar behavior. We survey the available results on graph distances in power-law random graphs that are consistent with this prediction. () 2008 American Institute of Physics. [DOI: 10.1063/1.2982927]
\end{abstract}

\section{INTRODUCTION}

In the past decade, many examples of real-world complex networks that are small worlds, scale-free, and highly clustered were found. In small worlds, graph distances are small, often less than or equal to 6 , for networks with several million vertices. In scale-free networks, degree sequences are power laws, irrespective of their size. A network is highly clustered when the neighbors of a vertex are more likely to be connected to each other than random pairs of vertices. See Refs. 2, 34, and 53 for reviews on complex networks and Ref. 5 for a more expository account. As a result, these complex networks are not at all like classical random graphs (see Refs. 3,8 , and 50 and the references therein) particularly since the classical model does not have power-law degrees. These empirical findings have ignited an enormous research on adaptations of the classical random graph that do obey power-law degree sequences. We shall survey some of these results in this article.

This paper is organized as follows. In Sec. II, we study three models for random graphs without geometry that can have rather general degrees, namely, inhomogeneous random graphs (IRGs), the configuration model (CM), and preferential attachment (PA) models. We survey results concerning the phase transitions in such models in Sec. III, and results on distances in such models in Sec. IV. Finally, in Sec. V, we treat some results on universality.

\section{THE MODELS}

Extensive discussions on scale-free random graphs can be found in Refs. 22 and 35. Durrett ${ }^{35}$ also investigated certain stochastic processes on random graphs, while Chung and $\mathrm{Lu}^{22}$ focused on random graphs with given expected degrees and also studied eigenvalue properties of the adja-

\footnotetext{
a)Electronic mail: rhofstad@win.tue.nl.

${ }^{\mathrm{b})}$ Electronic mail: g.hooghiemstra@ewi.tudelft.nl.
} 
cency matrix and related problems. We start by describing the models that we shall focus our discussion on in this survey, namely, IRGs, the CM, and affine PA models.

\section{A. Inhomogeneous Random Graphs}

The simplest random graph imaginable is the so-called Erdös-Rényi random graph, denoted by $G(n, p)$, which consists of $n$ vertices and each of the $n(n-1) / 2$ edges is occupied with probability $p$, independently of the occupation status of the other edges. This model was introduced by Gilbert, ${ }^{41}$ while Erdős and Rényi ${ }^{36}$ introduced a model where a fixed number of edges is chosen uniformly at random and without replacement. The two models are quite comparable, and asymptotic results in one of the two models can easily be transferred to the other. A model with a fixed number of edges being chosen with replacement, so that possibly multiple edges between vertices arise, can be found in Ref. 4. The name Erdős-Rényi random graph is given to this class of models due to the fact that the first rigorous results were derived in the seminal paper, ${ }^{37}$ which can be seen as having founded the field of random graphs, and which has inspired research question for decades after 1960 (see also the books ${ }^{8,50}$ ).

One of the charming features of the Erdös-Rényi random graph is the fact that its vertices are exchangeable. For example, each vertex $v \in[n]$, where we write $[n]=\{1, \ldots, n\}$ for the set of vertices, has a degree which is distributed as a binomial random variable with parameters $n-1$ and $p$. Thus, when $n p \rightarrow \infty$, the average degree tends to infinity, while for $n p=\lambda$, for some $\lambda$ $\in(0, \infty)$, the average degree remains uniformly bounded. It is this setting that we shall be mainly interested in since many real networks are sparse, i.e., they have a bounded average degree. When $p=\lambda / n$, the average degree of each vertex is roughly equal to $\lambda$, and the degree of a vertex converges in distribution to a Poisson random variable with parameter $\lambda$. It can be seen that also the proportion of vertices with degree $k$, i.e.,

$$
P_{k}^{(n)}=\frac{1}{n} \sum_{i=1}^{n} 1_{\left\{D_{i}(n)=k\right\}},
$$

where $D_{i}(n)$ is the degree of vertex $i$ in the graph of size $n$, converges in probability to the Poisson probability mass function $p_{k}=e^{-\lambda} \lambda^{k} / k$ ! as $n \rightarrow \infty$. Note that a Poisson distribution has quite thin, even subexponential, tails. For a graph of size $n$, we call the sequence $\left\{P_{k}^{(n)}\right\}_{k=0}^{\infty}$ the degree sequence of the graph.

In real-life networks, the degree sequence can be measured, and the empirical findings often show that the measured degree sequence resembles a power-law distribution, i.e., for large $n$, the estimate for $P_{k}^{(n)}$ is approximately proportional to $k^{-\tau}$ for some $\tau>1$. This power-law behavior is rather different from the Poisson probability mass function, and as a result, the Erdős-Rényi random graph is not a good model for many real networks. This problem can be overcome by taking the edge probabilities to be unequal instead of taking them constant. This yields the celebrated Inhomogeneous Random Graph, about which the seminal paper ${ }^{11}$ proves substantial results in full generality. See also Ref. 11 for several examples that were studied in literature, and which are generalized by Bollobás et al. ${ }^{11}$ We shall not go into the precise definition of the model in Ref. 11 but rather look at a simpler special case which allows for general degree sequences.

In the general setting of IRGs, we let $G(n, \mathbf{p})$ denote a general IRG, where $\mathbf{p}=\left\{p_{i j}\right\}_{1 \leq i<j \leq n}$ is such that $p_{i j}$ is the probability that the edge $i j=(i, j) \in[n]^{2}$ with $i \neq j$ is occupied, and where the occupation status of different edges are independent. The Erdős-Rényi random graph is retrieved when taking $p_{i j}=p$ for all edges $i j$. We now generalize the definition in such a way that power-law degrees can be obtained. We assign positive weights $\left\{w_{i}\right\}_{i=1}^{n}$ to the vertices, with $w_{i}$ as the weight of vertex $i$. Let $l_{n}=\sum_{i=1}^{n} w_{i}$ be the total weight. Then, we can take

$$
p_{i j}=1-e^{-w_{i} w_{j} / l_{n}} .
$$

In this way, we retrieve the Poisson random graph (PRG). ${ }^{54}$ Alternatively, for $p_{i j}$ $=\min \left\{w_{i} w_{j} / l_{n}, 1\right\}$, we retrieve the random graph with a given expected degree, as studied in detail 
by Chung and Lu. ${ }^{19-24}$ Note that, in this model, if we assume that $\max _{i=1}^{n} w_{i}^{2}<l_{n}$, and if we allow for a single self-loop at vertex $i$ with probability $w_{i}^{2} / l_{n}$, then the expected degree of vertex $i$ is precisely equal to $w_{i}$, which explains the name of this model. A third example occurs when we take $p_{i j}=w_{i} w_{j} /\left(l_{n}+w_{i} w_{j}\right)$, which is called the generalized random graph. ${ }^{17}$ In Ref. 48 conditions are given as to when two inhomogeneous graphs $G(n, \mathbf{p})$ and $G\left(n, \mathbf{p}^{\prime}\right)$ are equivalent, i.e., when events have asymptotically the same probability for $G(n, \mathbf{p})$ and $G\left(n, \mathbf{p}^{\prime}\right)$. In particular, in many cases, it can be seen that the expected degree random graph, the PRG, and the generalized random graph are equivalent, at least when the weights $\left\{w_{i}\right\}_{i=1}^{n}$ are not too large.

Since in an IRG the expected degree of vertex $i$ is close to $w_{i}$, in order to obtain a specified degree sequence in the graph, we need to pick the weights $\left\{w_{i}\right\}_{i=1}^{n}$ appropriately. In the sequel, we shall take

$$
w_{i}=w_{i}^{(n)}=[1-F]^{-1}(i / n),
$$

where $[1-F]^{-1}$ is the generalized inverse function of the survival function $x \mapsto 1-F(x)$ given by, for $u \in(0,1)$,

$$
[1-F]^{-1}(u)=\inf \{s:[1-F](s) \leq u\} .
$$

We call $G(n, \mathbf{p})$, with $\mathbf{p}$ as in (2) and $\left\{w_{i}\right\}_{i=1}^{n}$ as in (3) the rank-1 IRG with deterministic weights according to $F$. In this case, the degrees of $G(n, \mathbf{p})$ can be seen to have a mixed Poisson distribution with mixing distribution $F{ }^{17}$ i.e., the asymptotic probability that a uniform vertex has degree $k$ is

$$
q_{k}=\mathbb{E}\left[\frac{W^{k}}{k !} e^{-W}\right],
$$

where $W$ has a distribution function $F$. Alternatively, we can take $w_{i}=W_{i}$, where $\left\{W_{i}\right\}_{i=1}^{n}$ is an independent and identically distributed (i.i.d.) sequence of random variables with distribution $F$. We call $G(n, \mathbf{p})$ with $\mathbf{p}$ as in (2) and $\left\{W_{i}\right\}_{i=1}^{n}$ an i.i.d. sequence of random variables with distribution $F$, the rank-1 IRG with random weights according to $F$.

The advantage of random weights is that the vertices in the resulting graph are exchangeable; the disadvantage is that the edges are no longer independent, their dependence being moderated by the random weights.

\section{B. The Configuration Model}

We next define the CM. Let $\left\{d_{i}\right\}_{i=1}^{n}$ be a sequence of non-negative integer numbers. The integer $d_{i}$ will denote the degree of node $i$. Since for each graph the sum of degrees equals two times the number of edges, we demand that $\sum_{i=1}^{n} d_{i}$ is even. Without loss of generality, we may assume that $d_{i} \geq 1$ for all $i \in[n]$ since vertices $i$ with $d_{i}=0$ are isolated and may be removed from the graph.

For each $i \in[n]$, we attach $d_{i}$ half-edges to node $i$ and we pair the half-edges at random so that two half-edges will form one edge. From this construction, we obtain a random graph where multiple edges and self-loops are possible. However, for $n \rightarrow \infty$, self-loops and multiple edges are scarce. See, e.g., Ref. 47 for a proof that the number of self-loops and multiple edges converges to independent Poisson random variables, at least when $\sum_{i=1}^{n} d_{i}^{2} / n$ remains uniformly bounded. The paper ${ }^{47}$ gives the most general result in this direction and generalizes a substantial amount of previous work (see Ref. 47).

Similar to the rank-1 case of IRGs described above, there are two cases which were studied for the CM, depending on whether the degrees are deterministic or random. In the case of deterministic degrees, a possible choice to obtain a degree sequence with distribution function $F$ is to take $F$ as the distribution function of an integer random variable and to take the number of vertices with degree $k$ to be equal to $[n F(k)]-[n F(k-1)]$. We shall call this the $C M$ with deterministic degree according to $F$. For random degrees, we can take the degrees $\left\{D_{i}\right\}_{i=1}^{n}$ to be i.i.d. random variables with distribution function $F$, which we shall call the $C M$ with random degrees according 
to $F$. In the latter case, unless $D_{1}$ is even with probability 1 , the sum $\sum_{i=1}^{n} D_{i}$ can be seen to be odd with probability exponentially close to $1 / 2$. To avoid this problem, we can increase the degree of vertex $n$ by 1 so that $d_{n}=D_{n}+1$. This hardly makes any difference in the properties of the graph under consideration, and we will ignore this effect in the sequel.

\section{Preferential Attachment models}

While IRGs and the CM have power-law degree sequences when the edge probabilities or the degrees are chosen appropriately, they do not explain why many complex networks are scale-free. A possible explanation was given by Albert and Barabási ${ }^{6}$ by a phenomenon called Preferential Attachment. In PA models, the growth of the network is such that new vertices are more likely to attach their edges to vertices already having a high degree. For example, in social networks, a newcomer is more likely to get to know a person who is socially active and, therefore, already has a high degree. Interestingly, when the PA scheme is affine, as explained below, PA models have power-law degree sequences and, therefore, PA offers a convincing explanation why many realworld networks have power-law degree sequences. As a result, many papers appeared that study such models. See, e.g., Refs. 1, 9, 12-16, and 28 and references therein. The literature primarily focuses on three main questions. The first is to prove that such random graphs are indeed scale-free. ${ }^{1,9,12,13,16,28}$ The second is to show that the resulting models are small worlds by investigating the distances in them. See, for example, Ref. 15 for a result on the diameter. In nonrigorous work, it is often suggested that many of the scale-free models, such as the CM, the models in Ref. 11, and the PA models, have similar properties for their distances. Distances in the CM were shown to depend on the number of finite moments of the degree distribution. The natural question is therefore whether the same applies to PA models. A partial result in this direction is in Ref. 15. A third key question for PA models is their vulnerability, for example, to deliberate $\operatorname{attack}^{13}$ or to the spread of a disease. ${ }^{7}$

We now formulate a PA model, which is an extension of the Barabási-Albert model, as formulated rigorously first in Ref. 16 , by constructing the graph process $\left\{G_{m}(n)\right\}_{n=1}^{\infty}$. We start by formulating the model for $m=1$, for which we start with $G_{1}(1)$ consisting of a single vertex with a single self-loop. We denote the vertices of the graph by $1,2, \ldots$, so that the set of vertices of $G_{1}(n)$ is equal to $[n]$. We denote the degree of vertex $i$ at time $n$ by $D_{i}(n)$, where a self-loop increases the degree by 2 .

Then, for $m=1$, and conditionally on $G_{1}(n)$, the growth rule to obtain $G_{1}(n+1)$ is as follows. We add a single vertex $n+1$ having a single edge. This edge is connected to a second end point, which is equal to $n+1$ with probability proportional to $1+\delta$, and to a vertex $i \in G_{1}(n)$ with probability proportional to $D_{i}(n)+\delta$, where $\delta>-1$ is a parameter of the model. Thus,

$$
\mathbb{P}\left(n+1 \rightarrow i \mid G_{1}(n)\right)= \begin{cases}\frac{1+\delta}{n(2+\delta)+(1+\delta)} & \text { for } i=n+1 \\ \frac{D_{i}(n)+\delta}{n(2+\delta)+(1+\delta)} & \text { for } i \in[n] .\end{cases}
$$

The model with integer $m>1$ is defined in terms of the model for $m=1$ as follows. We take $\delta$ $>-m$, and then start with $G_{1}(m n)$, with $\delta^{\prime}=\delta / m>-1$, and denote the vertices in $G_{1}(m n)$ by $v_{1}^{(1)}, \ldots, v_{m n}^{(1)}$, while we denote the vertices of $G_{m}(n)$ by $v_{1}^{(m)}, \ldots, v_{n}^{(m)}$. Then we identify the vertices $v_{1}^{(1)}, v_{2}^{(1)}, \ldots, v_{m}^{(1)}$ in $G_{1}(m n)$ to be vertex $v_{1}^{(m)}$ in $G_{m}(n)$, and for $1<j \leq n$, the vertices $v_{(j-1) m+1}^{(1)}, \ldots, v_{j m}^{(1)}$ in $G_{1}(m n)$ to be vertex $v_{j}^{(m)}$ in $G_{m}(n)$; in particular, the degree $D_{j}(n)$ of vertex $v_{j}^{(m)}$ in $G_{m}(n)$ is equal to the sum of the degrees of the vertices $v_{(j-1) m+1}^{(1)}, \ldots, v_{j m}^{(1)}$ in $G_{1}(m n)$. This defines the model for integer $m \geq 1$. In the sequel, we shall denote the vertices in $G_{m}(m n)$ by $1, \ldots, n$ rather than $v_{1}^{(m)}, \ldots, v_{n}^{(m)}$, when no confusion can arise. The resulting graph $G_{m}(n)$ has precisely $m n$ edges and $n$ vertices at time $n$ but is not necessarily connected. For $\delta=0$, we obtain the original model studied in Ref. 16 and further studied in Refs. 13-15. The extension to $\delta \neq 0$ is crucial in our setting, as we shall explain in more detail below. The parameter $m$ denotes the initial 
degree with which new vertices enter, while the parameter $\delta>-m$ moderates the strength of the PA scheme. Indeed, when $\delta \rightarrow \infty$, each vertex is connected to with equal probability, while for $\delta$ $>-m$ close to $-m$, very rarely connections take place to vertices with minimal degree equal to $m$.

There are several related ways to define the model. For example, we can forbid self-loops when $m=1$ by setting the probability that $n+1$ connects to itself to be 0 , with intermediate updating of the degrees, or let the $m$ edges incident to vertex $n$ be attached independently without allowing vertex $n+1$ to connect to itself (see Ref. 43 for more details). For many of the results, this precise choice is irrelevant, and in this survey, we shall stick to the model in (6). The latter two versions have the nice feature that they both lead to connected random graphs.

We continue to discuss the degree sequence of the above PA model. Recall (1) for the definition of the degree sequence. Much of the available literature on PA models centers around the proof that the asymptotic degree sequence obeys a power law, where the exponent $\tau$ depends in a sensitive way on the parameters of the model. For the PA model considered here, the power-law exponent is equal to $\tau=3+\delta / m$, so that it can take any value $\tau \in(2, \infty)$, by adjusting the parameter $\delta>-m$. It is here that we rely on the choice of the model in (6), i.e., on the fact that our PA scheme is affine. A form of bias in growing networks toward vertices with higher degree is, from a practical point of view, quite likely to be present in various real networks, but it is unclear why the PA scheme should be affine as in (6). However, only affine PA schemes give rise to power-law degree sequences. See Refs. 55 and 57 for examples of PA models with (possibly) nonlinear PA mechanisms and their resulting degree sequences. In particular, Oliveira and Spencer ${ }^{55}$ and Rudas et al. ${ }^{57}$ showed that the degree sequence of a PA model depends sensitively on the details of the PA scheme.

We will now explain why the affine PA mechanism in (6) gives rise to power-law degree sequences and highlight the proof. Virtually, all proofs of asymptotic power laws in PA models consist of two key steps: one step where it is proved that the degree sequence is concentrated around its mean, and one where the mean degree sequence is identified. Let $N_{k}^{(n)}=n P_{k}^{(n)}$ be the number of vertices with degree $k$ in $G_{m}(n)$. We are interested in the limiting distribution of $P_{k}^{(n)}$ as $n \rightarrow \infty$. This distribution arises as the solution of a certain recursion, of which we will now give a short heuristic derivation. First note that, obviously,

$$
\mathbb{E}\left[N_{k}^{(n+1)} \mid G_{m}(n)\right]=N_{k}^{(n)}+\mathbb{E}\left[N_{k}^{(n+1)}-N_{k}^{(n)} \mid G_{m}(n)\right] .
$$

Asymptotically, for $n$ large, it is quite unlikely that a given vertex will be hit more than once by one of the $m$ edges added upon the addition of vertex $n$. Let us hence ignore this possibility for the moment. The difference $N_{k}^{(n+1)}-N_{k}^{(n)}$ between the number of vertices with degree $k$ at time $n+1$ and time $n$, respectively, is then obtained as follows.

(a) Vertices with degree $k$ in $G_{m}(n)$ that are hit by one of the $m$ edges emanating from vertex $n+1$ are subtracted from $N_{k}^{(n)}$. The conditional probability that a fixed edge is attached to a vertex with degree $k$ is approximately $(k+\delta) N_{k}^{(n)} /(n(2 m+\delta))$, so that (ignoring multiple attachments to a single vertex) the mean number of vertices to which this happens is approximately $m(k+\delta) N_{k}^{(n)} /(n(2 m+\delta))$. We note that we have replaced the numerator, which is actually equal to $n(2 m+\delta)+(e-1)(2+\delta / m)+1+\delta$ in the attachment of the $e$ th edge emanating from vertex $n$, by its approximate value $n(2 m+\delta)$ for large $n$.

(b) Vertices with degree $k-1$ in $G_{m}(n)$ that are hit by one of the $m$ edges emanating from vertex $n+1$ are added to $N_{k}^{(n)}$. By reasoning as above, it follows that the mean number of such vertices is approximately $m(k-1+\delta) N_{k}^{(n)} /(n(2 m+\delta))$.

(c) The new vertex $n+1$ should be added if it has a degree $k$. When we ignore the case that vertex $n$ attaches edges to itself, this happens precisely when $k=m$. 
Combining this gives

$$
\mathbb{E}\left[N_{k}^{(n+1)}-N_{k}^{(n)} \mid G_{m}(n)\right] \approx \frac{m(k-1+\delta)}{n(2 m+\delta)} N_{k-1}^{(n)}-\frac{m(k+\delta)}{n(2 m+\delta)} N_{k}^{(n)}+\mathbb{1}_{\{k=m\}} .
$$

Substituting (8) into (7) and taking expectations, we arrive at

$$
\mathbb{E}\left[N_{k}^{(n+1)}\right] \approx \mathbb{E}\left[N_{k}^{(n)}\right]+\frac{m(k-1+\delta)}{n(2 m+\delta)} \mathbb{E}\left[N_{k-1}^{(n)}\right]-\frac{m(k+\delta)}{n(2 m+\delta)} \mathbb{E}\left[N_{k}^{(n)}\right]+1_{\{k=m\}}
$$

Now assume that $P_{k}^{(n)}$ converges to some limit $p_{k}$ as $n \rightarrow \infty$, so that $N_{k}^{(n)} \sim n p_{k}$. Sending $n \rightarrow \infty$ and observing that $\mathbb{E}\left[N_{k}^{(n+1)}\right]-\mathbb{E}\left[N_{k}^{(n)}\right] \rightarrow p_{k}$, implies that $\mathbb{E}\left[N_{k}^{(n)} / n\right] \rightarrow p_{k}$, for all $k$, then yields the recursion

$$
p_{k}=\frac{m(k-1+\delta)}{2 m+\delta} p_{k-1}-\frac{m(k+\delta)}{2 m+\delta} p_{k}+1_{\{k=m\}} .
$$

By iteration, it follows that this recursion is solved by $p_{k}=0$ when $k<m$, and

$$
p_{k}=\frac{2+\delta / m}{k+2+\delta+\delta / m} \prod_{j=m+1}^{k} \frac{j-1+\delta}{j+2+\delta+\delta / m}, \quad k \geq m .
$$

It is not hard to see that, when $k \rightarrow \infty, p_{k} \sim c_{m} k^{-\tau}$, with $\tau=3+\delta / m$. This explains the occurrence of power-law degree sequences in affine PA models. The above argument can be made rigorous by showing that $\max _{k}\left|\mathbb{E}\left[N_{k}^{(n)}\right]-n p_{k}\right|$ remains uniformly bounded (see, e.g., Ref. 31).

In order to prove concentration of $N_{k}^{(n)}$, all proofs in literature make use of a clever martingale argument that was first used in Ref. 16. Define the Doob martingale $M_{t}$ by

$$
M_{t}=\mathbb{E}\left[N_{k}^{(n)} \mid G_{m}(t)\right], \quad t=0,1, \ldots, n .
$$

Then, $M_{0}=\mathbb{E}\left[N_{k}^{(n)}\right]$, while $M_{n}=N_{k}^{(n)}$, so that $N_{k}^{(n)}-\mathbb{E}\left[N_{k}^{(n)}\right]=M_{n}-M_{0}$. The key ingredient is the observation that, for all $t=1, \ldots, n,\left|M_{t}-M_{t-1}\right| \leq 2 m$ almost surely since the only vertices that are affected by the information of $G_{m}(t)$ instead of $G_{m}(t-1)$ are the vertices affected by the attachment of the edges incident to vertex $t$. Together, the concentration and the asymptotic mean give that $G_{m}(n)$ has an asymptotic degree sequence $\left\{p_{k}\right\}_{k \geq 1}$, where $p_{k}$ is close to a power law for $k$ large.

\section{A prediction of universality}

In nonrigorous studies, it is often suggested that various scale-free random graph models, such as the CM or various models with conditional independence of edges as in Ref. 11 behave in a similar way. One of the main reasons behind this belief is that most of such networks are locally trees, in the sense that loops are quite rare, and the available loops tend to be quite large. For example, Dorogovtsev et al. ${ }^{33}$ states that

"In these graphs and many others, small and finite loops (cycles) are rare and not essential, the architectures are locally treelike, which is a great simplifying feature extensively exploited. One may say, the existing analytical and algorithmic approaches already allow one to exhaustively analyze any locally treelike network and to describe cooperative models on it. Moreover, the tree ansatz works well even in numerous important situations for loopy and clustered networks."

Naturally, loops are present in most random graph models, and controlling them in a rigorous way is, in general, a difficult problem. However, the above ideas do give us an appealing prediction of the behavior in random graph models with certain degree sequences. More precisely, for power-law random graphs, this prediction can be formalized by conjecturing that the behavior on random graphs, such as the nature of the phase transition, distances in such graphs and the critical behavior of processes living on them, have the same behavior in graphs with the same power-law degree exponent. We shall discuss some of the results in this direction below. 
We start by investigating the phase transition on the above random graphs in Sec. III, and discuss the results on distances in random graphs in Sec. IV.

\section{PHASE TRANSITION IN RANDOM GRAPHS}

In this section, we study the phase transition in random graphs. The phase transition describes when the random graphs under consideration have a largest connected component or giant component which has size comparable to the number of vertices of the graph. Many real networks have a high degree of connectivity, and therefore, models in which such a giant component exists are most natural in applications. We first introduce some notation. For the configuration model with deterministic or random degrees according to $F$, we define

$$
\nu=\frac{\mathbb{E}[D(D-1)]}{\mathbb{E}[D]},
$$

where the random variable $D$ has a distribution function $F$. For the rank-1 IRG with deterministic or random weights according to $F$, we define

$$
\nu=\frac{\mathbb{E}\left[W^{2}\right]}{\mathbb{E}[W]},
$$

where now the random variable $W$ has distribution function $F$. For PA models, we let $\nu=m$. In the results below, we shall write that a sequence of events $\left\{E_{n}\right\}_{n \geq 1}$ occurs with high probability (whp) when $\lim _{n \rightarrow \infty} \mathrm{P}\left(E_{n}\right)=1$. It turns out that $\nu=1$ plays the role of a critical value for all these random graphs:

Theorem 3.1 (Phase transition in random graphs): [(a) and (b)] For the CM with deterministic or random degrees according to $F$, and for the rank-1 IRG with deterministic or random weights according to $F$, the largest connected component has, whp, size o $(n)$ when $\nu \leq 1$, and size $\zeta n(1+o(1))$, for some $\zeta>0$, when $\nu>1$, where $n$ is the size of the graph.

(c) For the PA model of size $n$, whp, the largest connected component has size o(n) when $\nu$ $=m=1$, while it is connected whp for $n \rightarrow \infty$ when $\nu=m>1$.

The result for IRG is a special case in Ref. 11, Theorem 3.1. Earlier versions for the random graph with given expected degrees appeared in Refs. 20 and 23 (see also the monograph ${ }^{22}$ ). For the CM, the first result in the generality of Theorem 3.1 appeared in Refs. 51 and 52 under stronger conditions than mentioned here. For the sharpest result see Ref. 49. The connectivity of PA models was investigated for $\delta=0$ in Ref. 15, it was extended to all $\delta>-m$ in Ref. 42, Chap. 11.

We now move on to study distances in random graphs. Naturally, it only makes sense to speak about distances in highly connected graphs, i.e., for graphs in which a giant component of size proportional to the size of the graph, exists. Thus, we shall restrict to $\nu>1$, for which Theorem 3.1 shows that a giant component exists.

\section{DISTANCES IN RANDOM GRAPHS}

In this section, we summarize the results on distances in power-law random graphs. We combine the results in the three models discussed in Sec. II by the value of their respective power-law exponent. We define $H_{n}$ to be the average distance in the graph of size $n$, i.e., the number of edges in the shortest path between two uniformly chosen connected vertices. Note that even in a fixed graph, $H_{n}$ is a random variable, as it depends on the uniformly chosen pair of vertices. We shall also discuss results on the diameter of the graph, which is the maximum of the shortest path distances between any pair of connected vertices. Both give information about distances in graphs, the average distance being a more robust and informative feature of the graph than the diameter. 


\section{A. Distances in graphs with finite variance degrees $(\tau>3)$}

The main results on distances in power-law random graphs with power-law exponent $\tau>3$ are summarized in the following theorem.

Theorem 4.1 (Distances in graphs with finite variance degrees): [(a) and (b)] For the CM and the rank-1 IRG of size $n, H_{n} / \log n$ converges in probability to $1 / \log \nu$, where $\nu$ is given by (13) for the CM and by (14) for the rank-1 IRG, when F in the definition of the models satisfies that there exist $c>0$ and $\tau>3$ such that

$$
1-F(x) \leq c x^{-(\tau-1)}, \quad x \geq 0 .
$$

(c) For the affine PA model of size $n$ with $\delta>0$, so that $\tau=3+\delta / m>3$, whp $H_{n} / \log n$ is bounded above and below by positive and finite constants.

The result for the rank-1 IRG can be found in Ref. 38, where it is also shown that the fluctuations of $H_{n}$ around $\log _{\nu} n$ remain bounded, both in the case of i.i.d. degrees as well as for deterministic weights under a mild further condition on the distribution function. The first result in this direction was proved in Refs. 19 and 21 for the expected degree random graph, in the case of admissible deterministic weights. We refer to Ref. 21, p. 94 for the definition of admissible degree sequences. The result for the CM can be found in Ref. 45 in the case of i.i.d. degrees, where again also the fluctuations around $\log _{\nu} n$ are determined. The result for deterministic degrees in the CM is conjectured in Ref. 38 but is not proved anywhere. We expect that the methodology in Ref. 45 can be simply adapted to this case. The result for the affine PA model was proved in Ref. 43, unfortunately the proof of convergence in probability is missing in this case. It would be of interest to identify the constant to which $H_{n} / \log n$ converges in this setting.

Since $\log n$ is much smaller than the size of the graph $n$, we can interpret Theorem 4.1 as saying that distances in large graphs are relatively small, thus quantifying the small-world paradigm. The asymptotics of distances of order $\log n$ is clearly also valid in finite trees of degree $r$ $\geq 3$, so that we can interpret Theorem 4.1 as saying that these models are alike trees with (average) degree $\nu+1$. Below, however, we shall see that distances can be even much smaller than $\log n$ when the degrees have infinite variance.

\section{B. Distances in graphs with finite mean, infinite variance degrees $(\tau \in(2,3))$}

When $\tau \in(2,3)$, the variance in the degrees becomes infinite, which is equivalent to the statement that, with $D_{i}(n)$ denoting the degree of vertex $i$ in the graph of size $n, \Sigma_{i=1}^{n} D_{i}(n)^{2}$ grows much faster than $n$. The following theorem shows that, in such cases, the distances are much smaller than $\log n$.

Theorem 4.2 (Distances in graphs with $\tau \in(2,3))$ : [(a) and (b)] For the CM with random degrees according to $F$ and the rank-1 IRG with deterministic weights according to $F$, $H_{n} / \log \log n$ converges in probability to $2 /|\log (\tau-2)|$ when the size of the graph $n \rightarrow \infty$, when $F$ in the definition of the models satisfies that there exist $c>0$ and $\tau \in(2,3)$ such that

$$
1-F(x)=c x^{-(\tau-1)}(1+o(1)), \quad x \rightarrow \infty .
$$

(c) For the affine PA model with $\delta<0$ and $m \geq 2$, so that $\tau=3+\delta / m \in(2,3), w h p, H_{n} / \log \log n$ is bounded above by a finite constant.

Theorem 4.2 shows that distances in infinite variance power-law graphs are ultrasmall, a notion invented in Ref. 27. For all practical purposes, we can think of $\log \log n$ as being a constant number, so distances in power-law random graphs with exponents $\tau \in(2,3)$ are almost bounded.

The result for the rank-1 IRG is proved in Refs. 19 and 21 for the expected degree random graph, in the case of deterministic weights $w_{i}=c(i / n)^{-1 /(\tau-1)}$, having average degree strictly greater than 1 and maximum degree $m$ satisfying $\log m \gg \log n / \log \log n$. These restrictions were lifted in Ref. 35, Theorem 4.5.2. Indeed, the bound on the average distance is not necessary since, for $\tau$ $\in(2,3), \nu=\infty$ and therefore the IRG is always supercritical. An upper bound as in Theorem 4.2[(a) and (b)] on average distances for the PRG was proved in Ref. 54.

In the case of i.i.d. degrees, the first result for the CM can be found in Reittu and Norros, ${ }^{56}$ 
who describe the distances in the core of the random graph, which consists of those vertices which have rather high degree. A simple branching process approximation can be used to show that from a uniform vertex, we can reach this core in a small number of steps. The result quoted here can be found in Ref. 46, where again also the fluctuations are determined and are proved to be bounded. The restrictions on $F$ in Ref. 46 are somewhat weaker than (16), as they also allow $x \mapsto x^{\tau-1}[1$ $-F(x)$ ] to be slowly varying under certain conditions on the regularly varying function. The results in Ref. 40 apply in this case as well, and show that, when the proportion of vertices with degrees 1 and 2 is positive, the diameter divided by $\log n$ converges to a positive constant. In Ref. 44 and for $\tau$ in $(2,3)$ it is shown that the diameter in the CM is bounded above by a constant times $\log \log n$ when there are no vertices of degree 1 and 2 . Thus, at least for the diameter, the details of the degree sequence (and not only its power-law exponent) matter.

The result for the affine PA model was proved in Ref. 43. Again, it would be of interest to identify the constant to which $H_{n} / \log \log n$ converges in this setting.

\section{Distances in graphs with infinite mean degrees $(\tau \in(1,2))$}

Only in the CM (Ref. 39) and the IRG (Ref. 31) it is possible that the power-law exponent $\tau$ of the distribution function $F$ of the degrees for the $\mathrm{CM}$ and of the weights for the rank-1 IRG satisfies $\tau \in(1,2)$. In general, this is not very realistic, as it means that either there are extremely many multiple edges (in the $\mathrm{CM}$ ) or the power-law exponent in the graph does not match the value of $\tau$ (in the IRG). Distances in the infinite mean case were studied in Ref. 39 and show that distances remain uniformly bounded by 3 . In Ref. 54 a similar result appears for the PRG. The intuition behind this is clear: all vertices are connected to vertices with very high degree, and these vertices form a complete graph so that distances are at most 3 .

\section{Conclusion on phase transition and distances}

The main tool in order to study the phase transition and distances in the CM and IRG is a comparison of the neighborhood of a vertex to a two-stage (multitype) branching process. Specifically, for the $\mathrm{CM}$, with deterministic or i.i.d. degrees, the branching process, which we denote by $\left\{Z_{k}\right\}_{k \geq 0}$, starts from $Z_{0}=1$, has offspring distribution $\left\{f_{n}\right\}_{n=1}^{\infty}$, where $f_{n}$ are the jump sizes of the distribution $F$, in the first generation, and offspring distribution

$$
g_{n}=\frac{(n+1) f_{n+1}}{\mathbb{E}[D]}, \quad n \geq 0,
$$

in the second and further generations. It is not hard to verify that the parameter $\nu$ in (13) is the expectation of the size-biased distribution $\left\{g_{n}\right\}_{n=0}^{\infty}$. For the rank-1 IRG with deterministic or random weights, the branching process $\left\{Z_{k}\right\}_{k \geq 0}$ has a mixed Poisson distribution with random parameter $W$ in the first generation and a mixed Poisson distribution with random parameter $W_{e}$, which has the size-biased distribution of $W$, in the second and further generations. Thus, when $W$ has a continuous density $w \mapsto f(w)$, the density of $W_{e}$ is equal to $f_{e}(w)=w f(w) / \mathbb{E}[W]$. It can be seen that these two mixed Poisson distributions are again related through (17), and that again the parameter $\nu$ in (14) equals the expectation of the size-biased distribution used as offspring distribution in the second and further generations. The condition $\nu>1$ assures that the branching process $\left\{Z_{k}\right\}_{k \geq 0}$ is supercritical, so that it can grow to a large size with positive probability (recall Theorem 3.1). Intuitively, all vertices for which the connected component is large (say larger than $n^{\varepsilon}$ for some $\varepsilon>0)$ are connected and thus form a single giant component. The constant $\zeta$ in Theorem 3.1[(a) and (b)] is the survival probability of the multitype branching process $\left\{Z_{k}\right\}_{k \geq 0}$.

In order to prove distance results, one then has to further investigate the growth of the number of vertices at a given distance using limit laws for branching processes. When (15) holds, the number of vertices at a given distance $k$ grows proportionally to $\nu^{k}$, which suggests that distances are of the order $\log _{\nu} n$, as stated in Theorem 4.1. When (16) holds, then, by the results of Davies, ${ }^{29}$ the growth is superexponential, i.e. $(\tau-2)^{k} \log \left(Z_{k}+1\right)$ converges almost surely to a limiting random variable $Y$, where $Y>0$ precisely when the branching process survives. Thus, conditionally 
on $Y=y>0$, the number of individuals in generation $k$ grows like $e^{(\tau-2)^{-k} y(1+o(1))}$ suggesting that distances are of order $\log \log n /|\log (\tau-2)|$. The factor 2 in Theorem 4.2 is due to the fact that in order for two vertices to meet, each of their neighborhoods needs to have size at least $n^{\varepsilon}$ for some $\varepsilon>0$.

When $\tau>3$, we see that the limiting distribution of the average distance in our rank-1 IRG model (see Ref. 38) and in the CM (see Ref. 45) are both described in terms of the martingale limit of $M_{k}=Z_{k} / \mathbb{E}\left[Z_{k}\right]$. While this martingale limit depends sensitively on the precise offspring distribution, even the dependence on these limits in the two models is the same. An explanation for this close resemblance is that the two models are quite closely related. Indeed, as proved in Ref. 17, the generalized random graph, conditioned on its degree sequence, is a uniform simple random graph with that degree sequence. On the other hand, the CM conditioned on simplicity is also a uniform simple random graph with that degree sequence, and the probability that the $\mathrm{CM}$ is simple remains uniformly bounded from below for $\tau>3$ (see, e.g., Ref. 47). While this does not prove the equivalence, it does explain it, even though we have never been able to make use of this fact in a more direct way. Indeed, the probability of the CM being simple remains strictly positive, but it does not converge to 1 , and, in order to investigate the fluctuations of the average distances, we are forced to investigate events of probabilities that do not converge to 0 or 1 . In particular, we do not know whether our results on fluctuations of the distances in Ref. 45 remain valid, with the same limiting distribution, when we condition the $\mathrm{CM}$ to be simple.

We complete this discussion by explaining a method of proof which has been fruitful for all three models in the regime where $\tau \in(2,3)$ to prove upper bounds on the graph distances of order $\log \log n$ (recall Theorem 4.2). When $\tau \in(2,3)$, there exist vertices with quite large degree (the maximal degree is of order $\left.n^{1 /(\tau-1)}\right)$, and these vertices or "hubs" can be effectively used to prove good upper bounds on distances. This argument has been applied to the IRG in Refs. 19, 21, and 54, to the CM with i.i.d. degrees in Refs. 44 and 56, and to the PA model in Ref. 43. We apply the argument to the rank-1 IRG with deterministic weights according to $F$, where $F$ satisfies that there exists $\tau \in(2,3)$ such that, for all $x \geq 1$,

$$
1-F(x) \geq x^{1-\tau} .
$$

We take $\sigma>1 /(3-\tau)$, and define the core of the IRG, Core $_{n}$, to be

$$
\text { Core }_{n}=\left\{i: w_{i} \geq(\log n)^{\sigma}\right\},
$$

i.e., the set of vertices with weight at least $(\log n)^{\sigma}$. Then, for every $\sigma>1 /(3-\tau)$, the diameter of Core $_{n}$ is, whp, bounded above by $2 \log \log n /|\log (\tau-2)|(1+o(1))$.

In order to obtain this bound, we let $u_{1}=n^{1 /(\tau-1)}(\log n)^{-1}$, and define

$$
\mathcal{N}^{(1)}=\left\{i \in[n]: w_{i} \geq u_{1}\right\} .
$$

Obviously, $\mathcal{N}^{(1)} \neq \varnothing$ since (3) and (18) together imply that $w_{1} \geq n^{1 /(\tau-1)}$. For some constant $C$ $>0$ sufficiently large and $k \geq 2$, we define recursively

$$
u_{k}=C\left(u_{k-1}\right)^{\tau-2} \log n \quad \text { and } \quad \mathcal{N}^{(k)}=\left\{i \in[n]: w_{i} \geq u_{k}\right\} .
$$

The definition of $u_{k}, k \geq 2$, is such that

$$
u_{k} u_{k-1}\left|\mathcal{N}^{(k-1)}\right| \approx u_{k} u_{k-1} n\left[1-F\left(u_{k-1}\right)\right] \geq C n \log n,
$$

this basic inequality will be used below.

By induction one can prove that for $k \in \mathbb{N}$,

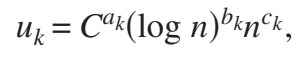

where 


$$
c_{k}=\frac{(\tau-2)^{k-1}}{\tau-1}, \quad b_{k}=\frac{1}{3-\tau}-\frac{4-\tau}{3-\tau}(\tau-2)^{k-1}, \quad a_{k}=\frac{1-(\tau-2)^{k-1}}{3-\tau} .
$$

In order to bound distances, we shall show that for each $k \geq 2$, the probability that there exists an $i \in \mathcal{N}^{(k)}$ that is not directly connected to $\mathcal{N}^{(k-1)}$ is $o\left(n^{-\gamma}\right)$, for some $\gamma>0$ independent of $k$.

To see this, let us consider the special case of the PRG, where the probability of no edges between node $i$ and node $j$ is given by $e^{-w_{i} w_{j} / l_{n}}$ (the other cases can be proved similarly). From Boole's inequality, the probability that there exists an $i \in \mathcal{N}^{(k)}$ such that $i$ is not directly connected to $\mathcal{N}^{(k-1)}$ is bounded by

$$
n \prod_{j \in \mathcal{N}^{(k-1)}} \exp \left\{-w_{i} w_{j} / l_{n}\right\} \leq n \exp \left\{-\frac{u_{k} u_{k-1} n\left[1-F\left(u_{k-1}\right)\right]}{l_{n}}\right\} \leq n \exp \left\{-\frac{C n \log n}{l_{n}}\right\},
$$

by (22). Since $l_{n}$ is of order $n$, this completes our claim.

We now complete the proof that the diameter of $\mathrm{Core}_{n}$ is, whp, bounded above by $2 \log \log n /|\log (\tau-2)|(1+o(1))$. Fix

$$
k^{*}=\left\lceil\frac{\log \log n}{|\log (\tau-2)|} \mid .\right.
$$

By the statement below (24), and since $k^{*} n^{-\gamma}=o(1)$, we have that whp every vertex in $\mathcal{N}^{(k)}$ is directly connected to a vertex in $\mathcal{N}^{(k-1)}$ for all $k \leq k^{*}$. Further, whp, all vertices in $\mathcal{N}^{(1)}$ are directly connected. We conclude that, whp, the diameter of $\mathcal{N}^{\left(k^{*}\right)}$ is at most $2 k^{*}+1$. Finally, in order to check that

$$
\operatorname{Core}_{n}=\left\{i: w_{i} \geq(\log n)^{\sigma}\right\} \subseteq \mathcal{N}^{\left(k^{*}\right)}=\left\{i: w_{i} \geq u_{k^{*}}\right\},
$$

we have to verify that $u_{k^{*}} \leq(\log n)^{\sigma}$ for any $\sigma>1 /(3-\tau)$. This can be done easily using the explicit value of $u_{k}$ given in (23) and the value of $k^{*}$ given in (26).

For the CM with deterministic degrees $d_{1}, d_{2}, \ldots, d_{n}$, where $d_{k}=[n F(k)]-[n F(k-1)]$, we change the definition of the core in (19), by replacing $w_{i}$ by $d_{i}$. An adaption of (25) then gives that also here, the diameter of Core ${ }_{n}$ is, whp, bounded above by $2 \log \log n /|\log (\tau-2)|(1+o(1))$.

For PA models, the proof is more involved, and we refer to Ref. 43 for the details. Recall that $\tau=3+\delta / m$, so that $-m<\delta<0$ corresponds to $\tau \in(2,3)$. Again we take $\sigma>1 /(3-\tau)$ and adapt the definition of the core to be

$$
\text { Core }_{n}=\left\{i \in[n]: D_{i}(n) \geq(\log n)^{\sigma}\right\},
$$

i.e., all the vertices which at time $n$ have degree at least $(\log n)^{\sigma}$. Then, by adapting the argument for the IRG given above, we can show that the diameter of Core ${ }_{n}$ in the PA model at time $2 n$ is, whp, bounded above by $4 \log \log n /|\log (\tau-2)|(1+o(1))$. A crucial role in the adaptation of this proof, which also explains the extra factor 2 in the upper bound on the diameter, is the notion of an $n$-connector. We say that $j \in[2 n] \backslash[n]$ is an $n$-connector between vertex $i \in[n]$ and a set of vertices $A \subset[n]$ if one of the edges incident to $j$ connects to $i$ and another connects to a vertex in $A$. In the proof for the PA model $n$-connectors play the same role as ordinary edges in the CM or IRG. When $j$ is an $n$-connector between $i$ and $A$, then the distance in $G_{m}(2 n)$ between $i$ and $A$ is at most 2. On the other hand, for the CM or the IRG, when $i$ is connected to $A$, then the distance between $i$ and $A$ is at most 1, meaning that distances in the PA model tend to be twice as large as for the CM and the IRG, at least in our upper bounds.

\section{DISCUSSION AND OPEN PROBLEMS}

The discussion on the phase transition and distances in random graphs has shown that the behavior in the IRG and in the CM is quite similar, as universality would predict, and that the neighborhoods of vertices in both models can be described in terms of branching processes. This 
is a powerful explanation of the appearing universality in the available distance results. For PA models, the picture is far less complete, predominantly since we do not understand the neighborhood structure of vertices too well. For $m=1$, a connection between general PA models and continuous-time branching processes is described in Ref. 57, where vertices attract new vertices at a rate that is a certain function of the degree of the receiving vertex. In fact, since vertices do not die, the resulting model is a pure birth process. The PA model of size $n$ is obtained as the branching process at the stopping time when the branching process has a total population $n$. Also, by definition, the PA model for $m \geq 2$ is obtained by collapsing or merging subsequent groups of $m$ vertices for the PA model with $m=1$ and $\delta^{\prime}=\delta / m$ into a single vertex. However, this merging or collapsing of vertices to go from the $m=1$ to the $m \geq 2$ case is a rather dramatic operation, and it is unclear whether the connection to continuous-time branching processes can be effectively used, for example, to study average distances.

An exciting question is how do stochastic processes on random graphs behave. Examples are abound: the spread of a disease in a population and a computer virus in the Internet, the spread of a rumor in a population, or how a population reaches consensus or cooperates. In all such questions, there is a delicate interplay between the structure or topology of the graph under consideration, and the properties of the stochastic process living on the graph. In the case of a random graph, this gives rise to a delicate double randomness, similar, for example, to the double randomness of a random walk in a random environment. While there is preliminary work on stochastic processes on random graphs, a general picture of the interplay between the topology of the graph and the stochastic process on it is still missing. It would be of great interest to investigate whether the behavior of stochastic processes on power-law random graphs is also universal in the sense that the critical behavior is determined by the power-law exponent $\tau$ of the degrees.

We now discuss some of the work on stochastic processes on random graphs. The problem which has received most attention is the vulnerability to random or deliberate attacks (see Refs. $11,13,25$, and 26). In random attacks, vertices are removed uniformly at random, while in deliberate attacks, the vertices with highest degrees are removed. This problem remains within the realms of random graph, as the resulting graph is again a random graph that may be studied with similar means as the original graph. However, even in this simplest problem, there is no complete picture of the relation between the structure of the original graph and the critical behavior of the graph remaining after the attack. The problem of the spread of a disease was studied for certain PA models in Ref. 7 and for the CM in Ref. 18. The problem of stochastic processes on random graphs has received substantial attention in the (nonrigorous) physics community, see, e.g., the extensive survey. ${ }^{33}$ It would be of great interest to clarify the picture of the dependence between the graph topology and the behavior of stochastic processes on them from a mathematical rigorous point of view. An example of particular interest from the point of view of statistical mechanics is the Ising model, a paradigm model for cooperative behavior on graphs. Preliminary results for the Ising model on the Erdős-Rényi random graph can be found in Refs. 10 and 30 and generalizations to graphs that are locally treelike in Ref. 32. The latter paper proves that the free energy exists in the $\tau>3$ regime for the CM and IRG.

${ }^{1}$ Aiello, W., Chung, F., and Lu, L., "Random evolution in massive graphs," Handbook of Massive Data Sets, Massive Computing Vol. 4 (Kluwer, Dordrecht, 2002), pp. 97-122.

${ }^{2}$ Albert, R. and Barabási, A.-L., "Statistical mechanics of complex networks," Rev. Mod. Phys. 74, 47 (2002).

${ }^{3}$ Alon, N. and Spencer, J., The Probabilistic Method, Series in Discrete Mathematics and Optimization, 2nd ed. (Wiley, New York, 2000).

${ }^{4}$ Austin, T. L., Fagen, R. E., Penney, W. F., and Riordan, J., "The number of components in random linear graphs," Ann. Math. Stat. 30, 747 (1959).

${ }^{5}$ Barabási, A.-L., Linked: The New Science of Networks (Perseus, Cambridge, Massachusetts, 2002).

${ }^{6}$ Barabási, A.-L. and Albert, R., "Emergence of scaling in random networks," Science 286, 509 (1999).

${ }^{7}$ Berger, N., Borgs, C., Chayes, J. T., and Saberi, A., On the Spread of Viruses on the Internet, Proceedings of the Sixteenth Annual ACM-SIAM Symposium on Discrete Algorithms (SIAM, Philadelphia, PA, 2005), pp. 301-310.

${ }^{8}$ Bollobás, B., Random graphs, Cambridge Studies in Advanced Mathematics Vol. 73, 2nd ed. (Cambridge University Press, Cambridge, England, 2001).

${ }^{9}$ Bollobás, B., Borgs, C., Chayes, J., and Riordan, O., Directed Scale-Free Graphs, Proceedings of the Fourteenth Annual ACM-SIAM Symposium on Discrete Algorithms (SIAM, Baltimore, MD, 2003), pp. 132-139. 
${ }^{10}$ Bollobás, B., Grimmett, G., and Janson, S., “The random-cluster model on the complete graph,” Probab. Theory Relat. Fields 104, 283 (1996).

${ }^{11}$ Bollobás, B., Janson, S., and Riordan, O., "The phase transition in inhomogeneous random graphs," Random Struct. Algorithms 31, 3 (2007).

${ }^{12}$ Bollobás, B. and Riordan, O., Handbook of Graphs and Networks (Wiley, Weinheim, 2003), pp. 1-34.

${ }^{13}$ Bollobás, B. and Riordan, O., "Robustness and vulnerability of scale-free random graphs," Internet Math. 1, 1 (2003).

${ }^{14}$ Bollobás, B. and Riordan, O., "Coupling scale-free and classical random graphs," Internet Math. 1, 215 (2004).

${ }^{15}$ Bollobás, B. and Riordan, O., "The diameter of a scale-free random graph," Combinatorica 24, 5 (2004).

${ }^{16}$ Bollobas, B., Riordan, O., Spencer, J., and Tusnády, G., "The degree sequence of a scale-free random graph process," Random Struct. Algorithms 18, 279 (2001).

${ }^{17}$ Britton, T., Deijfen, M., and Martin-Löf, A., "Generating simple random graphs with prescribed degree distribution," J. Stat. Phys. 124, 1377 (2006).

${ }^{18}$ Chatterjee, S. and Durrett, R., "Contact processes on random graphs with power law degree distributions have critical value 0," Preprint, 2008.

${ }^{19}$ Chung, F. and Lu, L., "The average distances in random graphs with given expected degrees," Proc. Natl. Acad. Sci. U.S.A. 99, 15879 (2002).

${ }^{20}$ Chung, F. and Lu, L., "Connected components in random graphs with given expected degree sequences," Ann. Comb. 6, 125 (2002)

${ }^{21}$ Chung, F. and Lu, L., "The average distance in a random graph with given expected degrees," Internet Math. 1, 91 (2003).

${ }^{22}$ Chung, F. and Lu, L., Complex Graphs and Networks, CBMS Regional Conference Series in Mathematics Vol. 107 (Conference Board of the Mathematical Sciences, Washington, DC, 2006).

${ }^{23}$ Chung, F. and Lu, L., "The volume of the giant component of a random graph with given expected degrees," SIAM J. Discrete Math. 20, 395 (2006).

${ }^{24}$ Chung, F., Lu, L., and Vu, V., "The spectra of random graphs with given expected degrees," Internet Math. 1, 257 (2004).

${ }^{25}$ Cohen, R., Erez, K., ben Avraham, D., and Havlin, S., "Resilience of the internet to random breakdowns," Phys. Rev. Lett. 85, 4626 (2000).

${ }^{26}$ Cohen, R. and Erez, K., ben Avraham, D., and Havlin, S., "Breakdown of the internet under intentional attack," Phys. Rev. Lett. 86, 3682 (2001).

${ }^{27}$ Cohen, R. and Havlin, S., "Scale-free networks are ultrasmall," Phys. Rev. Lett. 90, 058701 (2003).

${ }^{28}$ Cooper, C. and Frieze, A., "A general model of web graphs," Random Struct. Algorithms 22, 311 (2003).

${ }^{29}$ Davies, P. L., "The simple branching process: A note on convergence when the mean is infinite," J. Appl. Probab. 15, 466 (1978).

${ }^{30}$ De Sanctis, L. and Guerra, F., "Mean field dilute ferromagnet I: High temperature and zero temperature behavior," available at http://arxiv.org/abs/0801.4940v4

${ }^{31}$ Deijfen, M., van den Esker, H., van der Hofstad, R., and Hooghiemstra, G., "A preferential attachment model with random initial degrees," Ark. Mat. (to be published).

${ }^{32}$ Dembo, A. and Montanari, A., "Ising models on locally tree-like graphs," (unpublished).

${ }^{33}$ Dorogovtsev, S. N., Goltsev, A. V., and Mendes, J. F. F., "Critical phenomena in complex networks," available at http://arxiv.org/abs/0705.0010v6

${ }^{34}$ Dorogovtsev, S. N. and Mendes, J. F. F., "Evolution of networks," Adv. Phys. 51, 1079 (2002).

${ }^{35}$ Durrett, R., Random Graph Dynamics, Cambridge Series in Statistical and Probabilistic Mathematics (Cambridge University Press, Cambridge, England, 2007).

${ }^{36}$ Erdős, P. and Rényi, A., "On random graphs. I,” Publ. Math. (Debrecen) 6, 290 (1959).

${ }^{37}$ Erdős, P. and Rényi, A., "On the evolution of random graphs," Magy. Tud. Akad. Musz. Tud. Oszt. Kozl. 5, 17 (1960).

${ }^{38}$ van den Esker, H., van der Hofstad, R., and Hooghiemstra, G., "Universality for the distance in finite variance random graphs," J. Stat. Phys. (to be published).

${ }^{39}$ van den Esker, H., van der Hofstad, R., Hooghiemstra, G., and Znamenski, D., "Distances in random graphs with infinite mean degrees," Extremes 8, 111 (2005).

${ }^{40}$ Fernholz, D. and Ramachandran, V., "The diameter of sparse random graphs," Random Struct. Algorithms 31, 482 (2007).

${ }^{41}$ Gilbert, E. N., "Random graphs," Ann. Math. Stat. 30, 1141 (1959).

${ }^{42}$ van der Hofstad, R., "Random graphs and complex networks," see http://www.win.tue.nl/ rhofstad/NotesRGCN.pdf

${ }^{43}$ van der Hofstad, R. and Hooghiemstra, G., "Diameters in preferential attachment graphs," available at http://arxiv.org/ abs/0705.4153

${ }^{44}$ van der Hofstad, R., Hooghiemstra, G., and Znamenski, D., "A phase transition for the diameter of the configuration model," Internet Math. (to be published).

${ }^{45}$ van der Hofstad, R., Hooghiemstra, G., and Van Mieghem, P., "Distances in random graphs with finite variance degrees," Random Struct. Algorithms 27, 76 (2005).

${ }^{46}$ van der Hofstad, R., Hooghiemstra, G., and Znamenski, D. "Distances in random graphs with finite mean and infinite variance degrees," Electron. J. Probab. 12, 703 (2007).

${ }^{47}$ Janson, S., "The probability that a random multigraph is simple," available at http://arxiv.org/abs/math/0609.802

${ }^{48}$ Janson, S., "Asymptotic equivalence and contiguity of some random graphs," available at http://arxiv.org/abs/0802.1637

${ }^{49}$ Janson, S. and Luczak, M., "A new approach to the giant component problem," available at http://arxiv.org/abs/ 0707.1786

${ }^{50}$ Janson, S., Łuczak, T., and Rucinski, A., Random Graphs, Series in Discrete Mathematics and Optimization (Wiley, New York, 2000). 
${ }^{51}$ Molloy, M. and Reed, B., Proceedings of the Sixth International Seminar on Random Graphs and Probabilistic Methods in Combinatorics and Computer Science, "Random Graphs '93" (Poznań, 1993) Vol. 6, 1995 (unpublished), pp. 161179.

${ }^{52}$ Molloy, M. and Reed, B., "The size of the giant component of a random graph with a given degree sequence," Combinatorics, Probab. Comput. 7, 295 (1998).

${ }^{53}$ M. E. J. Newman. "The structure and function of complex networks," SIAM Rev. 45, 167, (2003).

${ }^{54}$ Norros, I., and Reittu, H., "On a conditionally Poissonian graph process," Adv. Appl. Probab. 38, 59 (2006).

${ }^{55}$ Oliveira, R. and Spencer, J., "Connectivity transitions in networks with super-linear preferential attachment," Internet Math. 2, 121 (2005).

${ }^{56}$ Reittu, H. and Norros, I., "On the power law random graph model of massive data networks," Perform. Eval. 55, 3 (2004).

${ }^{57}$ Rudas, A., Tóth, B., and Valkó, B., "Random trees and general branching processes," Random Struct. Algorithms 31, 186 (2007). 